\title{
Gain of Virulence on Rsv1-Genotype Soybean by an Avirulent Soybean mosaic virus Requires Concurrent Mutations in Both P3 and HC-Pro
}

\author{
A. L. Eggenberger, ${ }^{1}$ M. R. Hajimorad, ${ }^{2}$ and J. H. Hill ${ }^{1}$ \\ ${ }^{1}$ Department of Plant Pathology, lowa State University, Ames 50011 U.S.A.; ${ }^{2}$ Department of Entomology and Plant Pathology, \\ University of Tennessee, Knoxville 37996, U.S.A.
}

Submitted 4 February 2008. Accepted 1 April 2008.

In soybean, $R s v 1$, a single dominant resistance gene, invokes extreme resistance (ER) against most Soybean mosaic virus (SMV) strains, including SMV-N, but not SMV-G7, which provokes a virulent lethal systemic hypersensitive response (LSHR). The elicitor functions of the two viruses provoking Rsv1-mediated ER and LSHR have been mapped to the Nterminal 271 amino acids of $P 3$ from SMV-N and SMV-G7, respectively, which differ by nine residues between the two strains. To identify amino acids of $\mathrm{P3}$ from SMV-N provoking $R s v 1$-mediated ER, the unique residues of SMV-G7 were substituted with those of SMV-N. Of the mutants tested on Rsv1-genotype soybean, only SMV-G7 ${ }_{1788 R}$ and SMV-G7 ${ }_{\text {T948A }}$ lost virulence. However, substitution of amino acids of SMV-N, individually or in combination, with the reciprocal residues from SMV-G7 at these two positions failed to confer virulence to SMV-N. In the search for additional virulence determinants, a series of SMV-N chimeras was generated in which fragments within a region from near the middle of the helper-component proteinase (HCPro) cistron to the $5^{\prime}$ end of the cytoplasmic inclusion cistron, nucleotides 1,605 to 3,787 , were replaced with those of SMV-G7. Only SMV-N-derived chimeras harboring the $3^{\prime}$ region of $\mathrm{HC}$-Pro, at least from nucleotide 2,013 , and the entire $5^{\prime}$ end of $\mathrm{P3}$ (nucleotides 2,430 to 3,237 ) from SMVG7 were virulent whereas reciprocal exchanges resulted in loss of SMV-G7 virulence. This region of HC-Pro differs by three amino acids between SMV-N and SMV-G7. Analyses of SMV-G7-derived HC-Pro site-directed mutants showed that only SMV-G7 $7_{M 683 R}$ lost virulence on Rsv1-genotype soybean; however, $\mathrm{SMV}-\mathrm{N}_{\mathrm{R682M}}$ failed to gain virulence. Nevertheless, an SMV-N derived mutant with three concurrent substitutions, R682M+R787I+A947T, gained virulence. The data indicate that both $\mathrm{P3}$ and HC-Pro are involved in virulence of SMV on Rsv1-genotype soybean.

Additional keywords: Potyvirus, resistance response.

$R s v 1$, a single dominant resistance $(R)$ gene in soybean, confers resistance against Soybean mosaic virus (SMV) strains G1 to G6 but not G7 (Cho and Goodman 1979). Using SMV-N, a G2-strain isolate, the primary mechanism of resistance in me-

Corresponding author: M. R. Hajimorad; Fax: +1-865-974-4744; E-mail: mrh@utk.edu

* The $e$-Xtra logo stands for "electronic extra" and indicates a supplementary table and supplementary materials and methods information are published online. chanically inoculated $R s v 1$ leaves has been characterized as extreme resistance (ER), in which no symptoms are observed and virus cannot be recovered from the inoculated leaves. However, a restricted systemic hypersensitive response (SHR) is induced when SMV-N is introduced into Rsvl-genotype scions from infected rsvl-genotype soybean rootstocks (Hajimorad and Hill 2001). In contrast, SMV-G7 and its experimentally evolved variant, SMV-G7d (Hajimorad et al. 2003), are not limited in mechanically inoculated leaves by the Rsvl-mediated ER. SMV-G7 provokes a lethal SHR (LSHR) whereas SMV-G7d induces systemic mosaic (Hajimorad et al. 2003).

Analogous to the Potato virus X/Rxl pathosystem (Bendahmane et al. 1999), we hypothesized that the SMV-N elicitor of ER and restricted SHR are encoded by the same virus cistron, and the corresponding region of SMV-G7, albeit modified, is the elicitor of Rsv1-mediated LSHR (Hajimorad et al. 2005). By taking advantage of differential interactions of SMV-G7 and SMV-G7d with Rsv1-genotype soybean, the SMV-G7 elicitor of Rsv1-mediated LSHR was recently mapped to P3 and the amino acid residues involved were identified (Hajimorad et al. 2005). To demonstrate that P3 from a specific SMV strain is the elicitor of Rsvl-mediated ER, we also replaced the N-terminus or entire P3 of SMV-G7 or SMVG7d with those of SMV-N and vice versa, and compared the resultant chimeras for loss and gain of virulence on Rsv1genotype soybean (Hajimorad et al. 2006). Although all SMVG7- and SMV-G7d-derived P3 chimeras lost virulence on Rsv1-genotype soybean, neither of the SMV-N-derived P3 chimeras gained virulence (Hajimorad et al. 2006). These observations led us to conclude that P3 from specific SMV strains elicits Rsv1-mediated LSHR and ER; however, absence of the P3 elicitor function alone is insufficient for virulence on Rsv1genotype soybean (Hajimorad et al. 2005, 2006). Therefore, in addition to P3, SMV-G7 and SMV-G7d must have evolved additional genetic determinants for virulence on Rsvl-genotype soybean that reside elsewhere on the genome.

In search of additional virulence determinants of SMV on $R s v 1$-genotype soybean, we exploited the differential interactions of SMV-N and SMV-G7 on Rsvl-genotype soybean. In this report, we identify SMV-N P3 amino acids involved in elicitation of Rsv1-mediated ER; demonstrate that, in addition to $\mathrm{P} 3$, the $\mathrm{C}$-terminus of the helper-component proteinase (HC-Pro) is involved in the virulence of SMV on Rsvl-genotype soybean; and identify the amino acid involved. Moreover, we present evidence that concurrent mutations in the C-terminus of HC-Pro and the N-terminus of P3 of SMV-N are necessary and sufficient for virulence of SMV-N on Rsvl-genotype soybean.

Vol. 21, No. 7, 2008 / 931 


\section{RESULTS}

Two amino acids of $P 3$ from SMV-N independently convert virulent SMV-G7 to avirulent on Rsv1-genotype soybean.

We previously showed that replacement of the $5^{\prime}$ end (nucleotides 2,430 to 3,237 ) or entire P3 of SMV-G7 with sequences from SMV-N results in the loss of virulence of SMV-
G7 on Rsv1-genotype soybean (Hajimorad et al. 2006). This region of $\mathrm{P} 3$ differs between SMV-N and SMV-G7 by nine amino acids (Fig. 1A). To identify amino acids involved in elicitation of Rsvl-mediated ER, we replaced each of the unique amino acids of SMV-G7 with the corresponding amino acid from SMV-N. Analyses of the mutants on both rsvl-and $R s v 1$-genotype soybean showed that all were replication com-

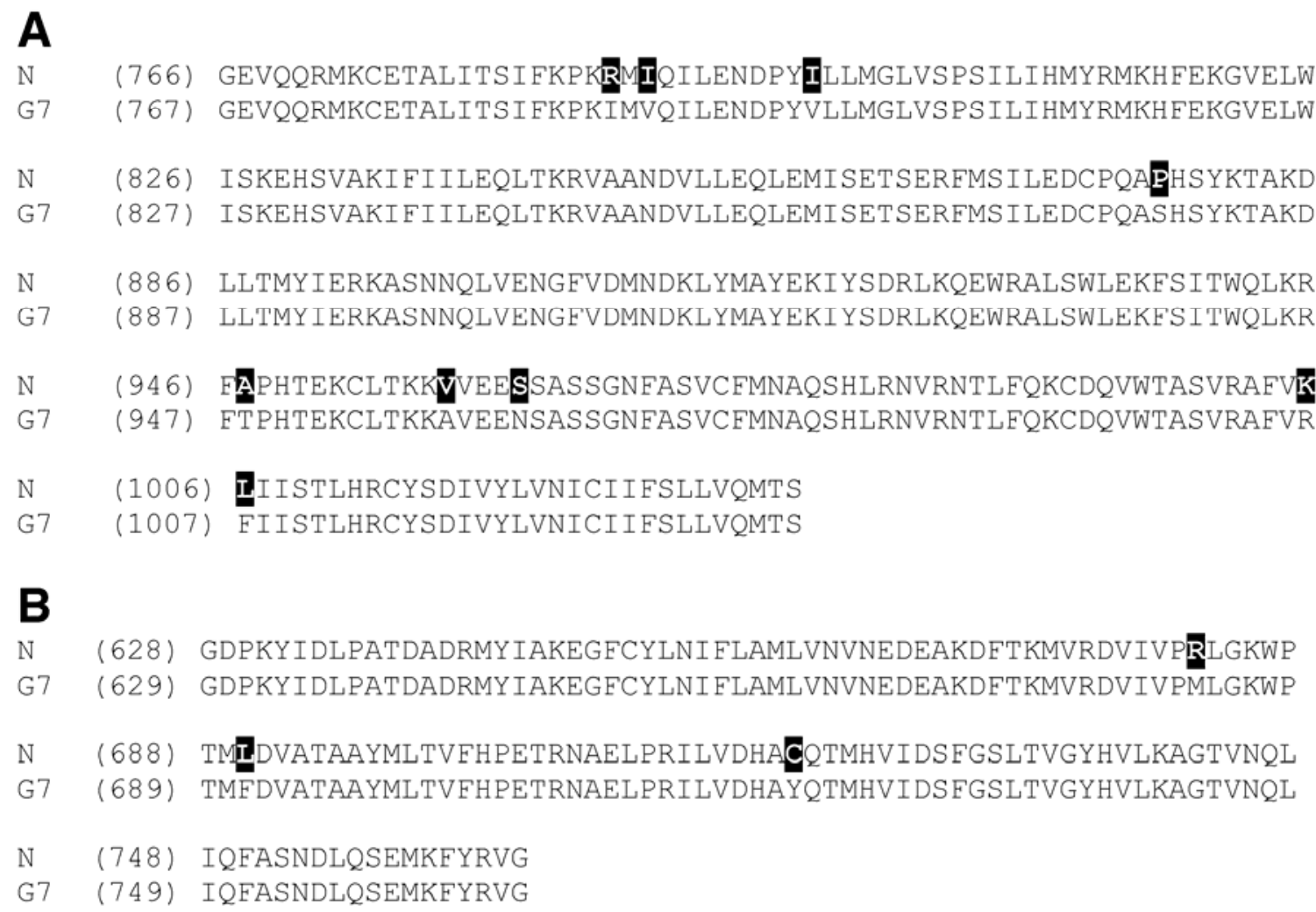

Fig. 1. Alignment of the A, P3 N-terminal and B, helper-component proteinase C-terminal amino acid sequences from Soybean mosaic virus (pSMV)-N (N) and pSMV-G7 (G7). The unique amino acids of N are highlighted in black. Note that amino acid positions for the SMV-N differ from that of SMV-G7 by one amino acid, because SMV-N lacks a codon in the P1 region of the genome (GenBank accession nos. D00507 and AY216010, respectively).

Table 1. Interaction of Soybean mosaic virus (SMV) strains N- or G7-derived mutants with rsv1 (Williams 82) and Rsv1 (L78-379) soybean ${ }^{\mathrm{a}}$

\begin{tabular}{|c|c|c|c|}
\hline \multirow[b]{2}{*}{ Mutant } & \multirow[b]{2}{*}{ Nucleotide substitution } & \multicolumn{2}{|c|}{ Soybean genotype } \\
\hline & & rsv1 (Williams 82) & Rsv1 (L78-379) \\
\hline SMV-G7 ${ }_{\text {M683R }}$ & $\mathrm{T} 2179 \mathrm{G}$ & + & - \\
\hline SMV-G7 F691L & C2204A & + & + \\
\hline SMV-G7 ${ }_{Y 721 C}$ & A2293G & + & + \\
\hline SMV-G7 I788R & T2494G & + & - \\
\hline SMV-G7 V790I & G2499A & + & + \\
\hline SMV-G7 V799I & $\mathrm{G} 2526 \mathrm{~A}$ & + & + \\
\hline SMV-G7 & $\mathrm{T} 2763 \mathrm{C}$ & + & + \\
\hline SMV-G7 T948A & A2973G & + & - \\
\hline $\mathrm{SMV}-\mathrm{G} 7_{\mathrm{A} 959 \mathrm{~V}}$ & $\mathrm{C} 3007 \mathrm{~T}$ & + & + \\
\hline SMV-G7 $7_{N 963 S}$ & A3019G & + & + \\
\hline SMV-G7 $7_{\text {R1006K }}$ & G3148A & + & + \\
\hline SMV-G7F1007L & T3150C & + & + \\
\hline SMV-N ${ }_{R 682 M}$ & $\mathrm{G} 2176 \mathrm{~T}$ & + & - \\
\hline SMV-N $\mathrm{R}_{\mathrm{R} 78 \mathrm{I}}$ & $\mathrm{G} 2491 \mathrm{~T}$ & + & - \\
\hline SMV-N $\mathrm{N}_{\mathrm{A} 947 \mathrm{~T}}$ & G2970A & + & - \\
\hline $\mathrm{SMV}-\mathrm{N}_{\mathrm{R} 682 \mathrm{M}+\mathrm{R} 787 \mathrm{I}}$ & $\mathrm{G} 2176 \mathrm{~T}+\mathrm{G} 2491 \mathrm{~T}$ & + & - \\
\hline $\mathrm{SMV}-\mathrm{N}_{\mathrm{R} 682 \mathrm{M}+\mathrm{A} 947 \mathrm{~T}}$ & $\mathrm{G} 2176 \mathrm{~T}+\mathrm{G} 2970 \mathrm{~A}$ & + & - \\
\hline SMV-N $\mathrm{R}_{\mathrm{R} 787 \mathrm{I}+\mathrm{A} 947 \mathrm{~T}}$ & $\mathrm{G} 2491 \mathrm{~T}+\mathrm{G} 2970 \mathrm{~A}$ & + & - \\
\hline SMV-N $\mathrm{R}_{682 \mathrm{M}+\mathrm{R} 787 \mathrm{I}+\mathrm{A} 947 \mathrm{~T}}$ & $\mathrm{G} 2176 \mathrm{~T}+\mathrm{G} 2491 \mathrm{~T}+\mathrm{G} 2970 \mathrm{~A}$ & + & + \\
\hline
\end{tabular}

a Plasmids containing full-length infectious cDNAs of mutants were delivered into expanded primary leaves of soybean seedlings by biolistic inoculation. The inoculated plants were maintained in a growth chamber $\left(22^{\circ} \mathrm{C}\right)$ and evaluated for the presence $(+)$ or absence $(-)$ of the viruses 6 weeks postinoculation by double-antibody sandwich enzyme-linked immunosorbent assay. The SMV-N helper-component proteinase cistron, nucleotides 1,056 to 2,426 , encodes amino acids 309 to 765 of the SMV-N polyprotein, whereas the SMV-N P3 cistron, nucleotides 2,427 to 3,623 encodes amino acids 766 to 1,164 of the polyprotein. Note that genomic positions for SMV-N differ from that of SMV-G7 by three nucleotides, because SMV-N lacks a codon in the P1 region. 
petent in rsv1-genotype soybean; however, SMV-G7 $7_{\mathrm{I788R}}$ and SMV-G7 ${ }_{\text {T948A }}$ lost virulence on Rsv1-genotype soybean (Table 1). Interestingly, when amino acids from SMV-N were replaced at these two locations with the corresponding amino acids from SMV-G7 $\left(\mathrm{N}_{\mathrm{R} 787 \mathrm{I}}\right.$ and $\mathrm{N}_{\mathrm{A} 947 \mathrm{~T}}$ ) (Table 1), individually or in combination, none of the resultant mutants gained virulence on $R s v 1$-genotype soybean; however, both remained replication competent in $r s v 1$-genotype soybean (Table 1).

\section{SMV-N-derived chimeras containing the $\mathrm{C}$-terminus of HC-Pro and the N-terminus of P3 from SMV-G7 gain virulence on Rsv1-genotype soybean.}

In order to identify other determinants of SMV-G7 necessary for virulence on $R s v 1$-genotype soybean, we generated a series of pSMV-N-derived chimeras where fragments within a region from near the middle of the HC-Pro cistron to the $5^{\prime}$ end of the cytoplasmic inclusion cistron (CI), nucleotides 1,605 to 3,782, were replaced with those from SMV-G7 (Fig. 2). All SMV-Nderived chimeras were replication competent in $r s v 1$-genotype soybean (Fig. 2). However, on Rsvl-genotype soybean, only SMV-N-derived chimeras containing both the C-terminal onethird of HC-Pro and the N-terminal two-thirds of P3 from SMV-G7 were virulent (Fig. 2). Virulence determinants of SMV-G7 in the HC-Pro cistron were encoded by a region from nucleotides 2,016 to 2,429 and those on P3 were encoded by a region from nucleotides 2,430 to 3,237 (Fig. 2). Replacement of homologous regions of SMV-G7 with a fragment containing these regions from pSMV-N resulted in loss of virulence of SMV-G7/N $\mathrm{N}_{(2013-3234)}$ on Rsv1-genotype soybean (note that the nucleotide numbering of HC-Pro and P3 cistrons differs between SMV-N and SMV-G7 because of a one-codon deletion in P1 of SMV-N). However, it remained replication competent in rsv1-genotype soybean (Fig. 2). Both SMV-N/G7 (2016-2429) and SMV-N/G7 ${ }_{(2338-3237)}$ remained avirulent on Rsv1-genotype soybean (Fig. 2). Interestingly, SMV-N/G7 ${ }_{(1608-2429,2828-3237)}$ remained avirulent on Rsvl-genotype soybean (Fig. 2). This chimera contains threonine at amino acid 948 of SMV-G7 but not isoleucine at residue 787 of SMV-N, which suggests that both SMV-N R787I and A947T (Table 1) are essential for HC-Pro complementation of virulence of SMV-N on Rsvl-genotype soybean.

\section{Substitution of a single amino acid of HC-Pro from SMV-G7 with that of SMV-N results} in loss of virulence on Rsv1-genotype soybean.

The C-terminus of HC-Pro (encoded by nucleotides 2,016 to 2,429 of SMV-G7) differs between SMV-N and SMV-G7 by three amino acids (Fig. 1B). To identify which unique amino acid of SMV-G7 is involved in virulence on $R s v 1$-genotype soybean, we replaced each residue with that of SMV-N

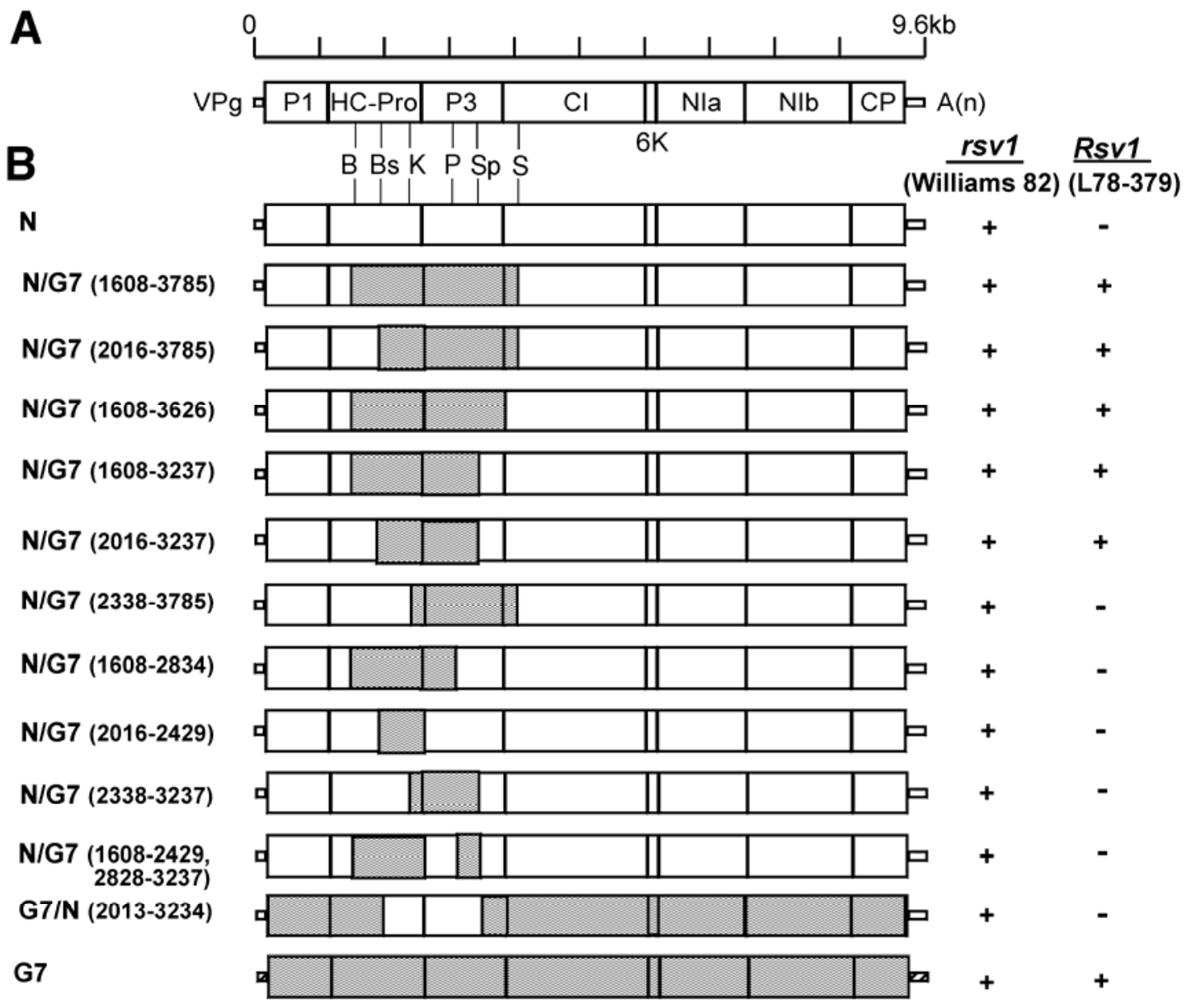

Fig. 2. Schematic representation of parental viruses or their derivative chimeras and their potential to infect $r s v 1$ (Williams 82 ) or Rsv1 (L78-379) soybean genotypes. A, The genomic map of Soybean mosaic virus (SMV) and the position of the restriction sites BglII (B), BstEII (Bs), KpnI (K), PflMI, (P), SpeI (Sp), and SalI (S) common between SMV-N and SMV-G7. B, Schematic representation of pSMV-N (N), and pSMV-G7 (G7), and their derivative chimeras with the response of Williams 82 ( $r s v 1)$ and L78-379 (Rsv1) to biolistic inoculation of plasmids and mechanical inoculation with progenies derived from the plasmids, respectively. Following inoculation, the plants were maintained in a growth chamber $\left(22^{\circ} \mathrm{C}\right)$ until evaluated 6 weeks post inoculation for the presence $(+)$ or absence $(-)$ of virus by double-antibody sandwich enzyme-linked immunosorbent assay. Note that genomic positions for SMV-N differ from that of SMV-G7 by three nucleotides, because SMV-N lacks a codon in the P1 region (GenBank accession nos. D00507 and AY216010, respectively). 
(Table 1). All three mutants (SMV-G7 $7_{M 683 R}, \mathrm{SMV-G} 7_{\mathrm{F} 6911}$, and SMV-G7 $\left.7_{\mathrm{Y} 721 \mathrm{C}}\right)$ were replication competent in $r s v 1$-genotype soybean (Table 1). However, on Rsvl-genotype soybean, only $\mathrm{SMV}-\mathrm{G} 7_{\mathrm{M} 683 \mathrm{R}}$ lost virulence. Interestingly, when the amino acid of SMV-N at this position was replaced with that of SMV-G7, $\mathrm{SMV}-\mathrm{N}_{\mathrm{R} 682 \mathrm{M}}$ remained replication competent in rsvl-genotype soybean but did not gain virulence on Rsvl-genotype soybean (Table 1).

\section{Gain of virulence by SMV-N on Rsv1-genotype soybean requires concurrent mutations in both $\mathrm{HC}$-Pro and $\mathrm{P3}$.}

Both SMV-G7 $7_{1788 \mathrm{R}}$ and SMV-G7 $7_{\text {T948A }}$ lost virulence on Rsv1genotype soybean (Table 1); however, SMV-N $\mathrm{R}_{\mathrm{R} 787 \mathrm{I}}$ and SMV$\mathrm{N}_{\mathrm{A} 947 \mathrm{~T}}$ failed to gain virulence. Furthermore, SMV- $\mathrm{N}_{\mathrm{R} 682 \mathrm{M}}$ remained avirulent on $R s v l$-genotype soybean. To determine whether the R682M mutation in HC-Pro can complement each of the mutations in $\mathrm{P} 3$ for virulence on Rsv1-genotype soybean, SMV-N $\mathrm{N}_{\mathrm{R} 682 \mathrm{M}+\mathrm{R} 787 \mathrm{I}}$ and $\mathrm{SMV}-\mathrm{N}_{\mathrm{R} 682 \mathrm{M}+\mathrm{A} 947 \mathrm{~T}}$ were constructed. Both SMV-N double mutants remained replication competent in $r s v 1$-genotype soybean but did not gain virulence on $R s v 1$ genotype soybean. SMV-N became infectious on $R s v 1$-genotype soybean only when all three mutations (R682M+R787I+A947T) were combined, resulting in induction of an LSHR similar to that produced by SMV-G7 (Fig. 3). The HC-Pro and P3 cistrons were recovered and sequenced. The absence of any other mutation in progenies from Rsvl-genotype soybean was confirmed.

\section{DISCUSSION}

In this report, we exploited differential interactions of SMV-N and SMV-G7 with Rsvl-genotype soybean and, through construction of a series of chimeras and site-directed mutants, mapped the avirulence determinants of SMV-N to one amino acid residue within the $\mathrm{C}$-terminus of $\mathrm{HC}$-Pro and two residues within the N-terminus of P3. In the context of SMV-G7, individual replacement of any of these amino acids with the corresponding residue of SMV-N resulted in loss of virulence on Rsvl-genotype soybean. However, in the context of SMV-N, individual reciprocal exchanges did not alter the avirulence of the virus and concurrent replacement of all three amino acids was required to restore virulence. The findings that two amino acids within the N-terminus of P3 from SMV-N converted SMV-G7 to avirulent on Rsv1-genotype soybean and reciprocal exchanges had no effect on the avirulence of SMV-N are consistent with our earlier report (Hajimorad et al. 2006).

Based on the above finding that all the three mutations would have to occur almost simultaneously for SMV-N to

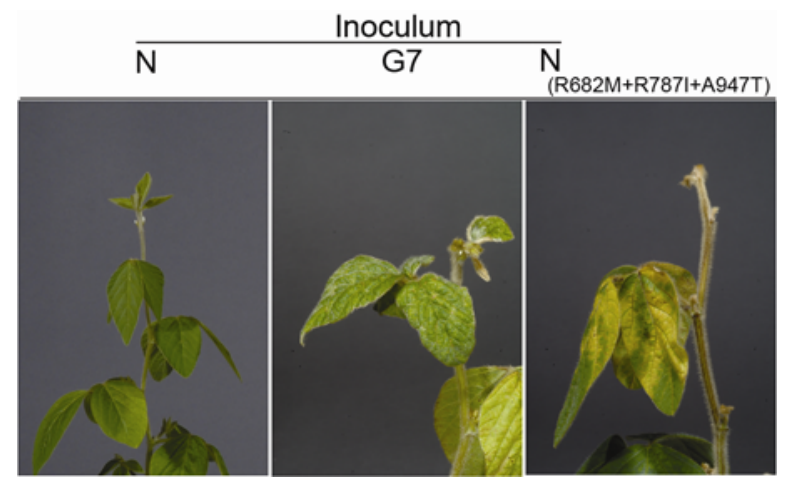

Fig. 3. Phenotypic response of soybean line L78-379 (Rsv1) to biolistic inoculation of Soybean mosaic virus (pSMV)-N (N), pSMV-G7 (G7), or a pSMV-N-derived mutant containing triple mutations (pSMV-

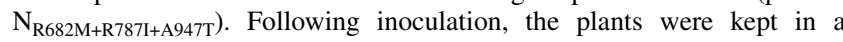
growth chamber $\left(22^{\circ} \mathrm{C}\right)$ until photographed 8 weeks post inoculation. evade ER mediated by $R s v 1$, it is predicted that $R s v 1$ would be durable against SMV-N and similar isolates. The avirulence determinants are stably maintained in the absence of Rsvl (unpublished data), suggesting a fitness cost for their loss similar to that observed for the avirulence determinants of the UK1 isolate of Turnip mosaic virus (TuMV) recognized by TuRBO1 from Brassica napus (Jenner et al. 2002b).

The mutations that conferred avirulence to SMV-G7 on Rsv1-genotype soybean, M683R, I788R, and T948A, are not predicted to significantly alter the secondary structure of the HC-Pro and P3 proteins. This is consistent with the observation that all three mutants were replication competent in Williams 82 ( $r s v 1)$ and suggests that the tertiary structure also was not significantly affected. Changes in the amino acid properties more likely affect protein-protein interactions. The mutations M683R in HC-Pro and I788R in P3 both result in changes from hydrophobic amino acids to positively charged amino acids, whereas the T948A mutation results in a change from a polar to a hydrophobic amino acid.

The involvement of two cistrons of a Potyvirus sp. in overcoming $R$-gene-mediated resistance responses in a single host has been reported. TuMV requires mutations in both P3 and CI to overcome the resistance response in B. napus line 165 (Jenner et al. 2002a). In this case, however, the involvement of two independent TuMV-encoded proteins was attributed to the existence of two independent $R$ genes against the virus in the host (Jenner et al. 2002a). It is interesting to note that the genetic determinant of another Potyvirus sp., Tobacco etch virus eliciting vascular necrosis in Tabasco pepper, was mapped to two separate regions in the genome and alterations of both regions was found essential for avoiding the necrosis response (Chu et al. 1997). Similarly, the viral determinants of a Lettuce mosaic virus strain, inducing systemic wilting in some lettuce cultivars, was recently mapped to the P1 and CI cistrons of the virus (Krause-Sakate et al. 2005). However, unlike Rsv1-genotype soybean, the genetic basis of vascular necrosis in Tabasco pepper and systemic wilting in the lettuce cultivars has not been determined and it is not known if an $R$ gene is involved (Chu et al. 1997; Krause-Sakate et al. 2005).

The observation that SMV-N $\mathrm{N}_{\mathrm{R} 682 \mathrm{M}+\mathrm{R} 787 \mathrm{I}+\mathrm{A} 947 \mathrm{~T}}$ was virulent upon direct biolistic inoculation of infectious cDNA onto unifolate leaves of Rsvl-genotype soybean suggests that HC-Pro is the only cistron in addition to P3 that is involved. In an independent study, Hajimorad and associates (2008) have demonstrated that experimental adaptation of a number of avirulent SMV-N-derived chimeras containing P3 sequences from two virulent SMV-strains G7 and G7d (Hajimorad et al. 2003) to Rsvl-genotype soybean also requires mutations in HC-Pro. Interestingly, they found that adaptation of one of the SMV-Nderived chimeras containing the N-terminus of P3 of SMV-G7 was associated with a mutation in HC-Pro at amino acid 682; however, the mutation was to a glycine residue. It seems clear that the SMV elicitor of $R s v 1$-mediated defense responses is limited to the P3 and HC-Pro cistrons; however, their functional roles in interaction with the Rsvl-genotype soybean remain to be understood.

The fact that both HC-Pro and P3 are involved in virulence of SMV-G7 on Rsv1-genotype soybean raises questions with regard to the nature of SMV elicitor of Rsvl and the mechanism of $R s v 1$-mediated recognition of SMV. It is not known whether HC-Pro and P3 are recognized as individual proteins or as some form of a precursor polyprotein. The genome of SMV, similar to any other Potyvirus sp., consists of a single open reading frame that codes for a single polypeptide that is cleaved by three virus-encoded proteinases to produce 8 to 10 mature proteins (Hull 2002; Jayaram et al. 1992). The presence of P3 in intermediates of polyprotein processing has been 
demonstrated for another Potyvirus sp., Tobacco mottling virus (Rodriguez-Cerezo and Shaw 1991). It also is not known whether the SMV-N proteins are recognized directly or indirectly by the Rsv1 protein. Because HC-Pro and P3 are unrelated at the sequence level, it may be that they interact with a common soybean factor, which is "guarded" (Dangl and Jones 2001) by $R s v 1$, analogous to the indirect recognition of the unrelated avirulence determinants $A v r B$ and AvrRpml by RPMI in Arabidopsis thaliana (Mackey et al. 2002).

Although high-resolution mapping has provided evidence that a single gene was associated with the Rsvl phenotype, the possibility that one of the other five nucleotide-binding-site leucine-rich-repeat genes in the tightly linked gene cluster also recognizes SMV has not been excluded (Hayes et al. 2004). Future work will explore this possibility as well as characterization of the interaction between the Rsv1 protein and the SMV-N HC-Pro and P3.

\section{MATERIALS AND METHODS}

\section{Virus isolates and soybean cultivars.}

Plasmids containing infectious full-length cDNA clones of SMV-N (pSMV-N) and SMV-G7 (pSMV-G7) proximal to the Cauliflower mosaic virus $35 \mathrm{~S}$ promoter propagated in $\mathrm{DH} 5 \alpha$ served as virus sources (Hajimorad et al. 2003; Wang et al. 2006). The plasmids were purified using a QiaPrep Spin MaxiPrep kit (Qiagen, Valencia, CA, U.S.A.). The soybean (Glycine max) cv. Williams 82 (rsvl) (Bernard and Cremeens 1988), susceptible to all strains of SMV, and line L78-379 (Rsv1) (Buzzell and Tu 1984), which is a near isoline of Williams (Bernard and Lindahl 1972) with the $R s v 1$ allele derived from line plant introduction (PI) 96983 [Williams (6) $\times$ PI 96983] (Bernard et al. 1991), were used in this study. All seed were obtained from plants shown to be free of SMV by indexing. Plants were grown in a growth chamber at $22^{\circ} \mathrm{C}$ with a photoperiod of $16 \mathrm{~h}$ or in a greenhouse with supplemental lighting.

\section{Construction of SMV chimeras and site-directed mutants.}

Most chimeras were generated by exchanging restriction fragments between cDNAs using standard molecular biological methods (Sambrook and Russell 2001). The restriction sites used and their nucleotide positions in the SMV-N genome were BglII (1,605), BstEII (2,013), KpnI (2,335), PflMI, (2,831), SpeI $(3,234)$, and SalI $(3,782)$ (Fig. 2). The genomic positions for these restriction sites on SMV-G7 differ from those of SMV-N by three nucleotides, because SMV-N lacks a codon relative to $\mathrm{SMV}-\mathrm{G} 7$ in the $\mathrm{P} 1$ region. Two chimeras, pSMV-N/G7 ${ }_{(2016-2429)}$ and pSMV-N/G7 ${ }_{(1608-3626)}$ (Fig. 2), were constructed to make the $\mathrm{pSMV-G7} \mathrm{sequence} \mathrm{terminate} \mathrm{precisely}$ at the end of the HC-Pro and P3 cistrons, respectively. Point mutations were constructed by performing an initial polymerase chain reaction (PCR) with the mutagenic primer and a primer $3^{\prime}$ of the region to be cloned. That PCR product was then used as a megaprimer in a PCR with a primer $5^{\prime}$ of the region to be cloned. Oligonucleotides used for creating point mutations and certain chimeras are listed in Supplementary Table 1. Details of creating point mutations and certain chimeras using PCR are listed in the Supplementary Materials and Methods.

\section{Inoculation of plants and detection of SMV.}

Virus infection was initiated by biolistic delivery into hypocotyls of Williams 82 (rsv1) (Hajimorad et al. 2003) or into expanded primary leaves of seedlings of Williams $82(r s v 1)$ and L78-379 (Rsvl) (Wang et al. 2006). Viral progenies were transferred by mechanical inoculation onto soybean leaves dusted with 600-mesh Carborundum (Hajimorad and Hill 2001). SMV was detected by double-antibody sandwich enzyme-linked immunosorbent assay (Hajimorad and Hill 2001).

\section{Reverse-transcriptase PCR, PCR, and sequencing.}

Reverse-transcriptase (RT)-PCR was performed using RNA PCR 2.1 and 3.0 kits (Takara Bio, Madison, WI, U.S.A.). Templates for reverse transcription were immunocaptured virus (Wang et al. 2006) or total plant RNA prepared using an RNeasy Plant Mini Kit (Qiagen). Primers for reverse transcription were random 9-mers (Takara Bio). PCR for constructing point mutations was performed using LA Taq DNA polymerase (Takara Bio). All PCR-amplified regions of plasmids were sequenced to verify that only the desired sequences were present. Sequencing was done at the Iowa State University DNA Facility. The identity of virus from infected plants and stability of the mutants was confirmed by sequencing of RT-PCR products.

\section{Protein structure analyses.}

Predictions of protein secondary structures were performed using the Wisconsin Package Version 10.2 (Genetics Computer Group, Madison, WI, U.S.A.) and the PredictProtein server (Rost et al. 2004).

\section{ACKNOWLEDGMENTS}

This project was funded in part by the Iowa Soybean Association, the North Central Soybean Research Program, and the University of Tennessee College of Agriculture and Experimental Station. This journal paper of the Iowa Agriculture and Home Economics Experiment Station, Ames, IA, project no. 3608, was supported, in part, by Hatch Act and State of Iowa funds. We thank M. Mazarei (University of Tennessee) and K. L. Perry (Cornell University) for critical reading of the manuscript.

\section{LITERATURE CITED}

Bendahmane, A., Kanyuka, K., and Baulcombe, D. C. 1999. The $R x$ gene from potato controls separate virus resistance and cell death responses. Plant Cell 11:781-791.

Bernard, R. L., and Cremeens, C. R. 1988. Registration of "Williams 82" soybean. Crop Sci. 28:1027-1028.

Bernard, R. L., and Lindahl, D. A. 1972. Registration of Williams soybean. Crop Sci. 12:716.

Bernard, R. L., Nelson, R. L., and Cremeens, C. R. 1991. USDA soybean genetic collection: isoline collection. Soybean Genet. Newsl. 18:27-57.

Buzzell, R. I., and Tu, J. C. 1984. Inheritance of soybean resistance to soybean mosaic virus. J. Hered. 75:82.

Cho, E.-K., and Goodman, R. M. 1979. Strains of soybean mosaic virus: classification based on virulence in resistant soybean cultivars. Phytopathology 69:467-470.

Chu, M., Lopez-Moya, J. L., Llave-Correas, C., and Pirone, T. P. 1997. Two separate regions in the genome of the tobacco etch virus contain determinants of the wilting response of Tabasco pepper. Mol. PlantMicrobe Interact. 10:472-480.

Dangl, J. L., and Jones, J. D. C. 2001. Plant pathogens and integrated defence responses to infection. Nature 411:826-833.

Hajimorad, M. R., and Hill, J. H. 2001. Rsv1-mediated resistance against Soybean mosaic virus- $\mathrm{N}$ is hypersensitive response-independent at inoculation site, but has the potential to initiate a hypersensitive responselike mechanism. Mol. Plant-Microbe Interact. 14:587-598.

Hajimorad, M. R., Eggenberger, A. L., and Hill, J. H. 2003. Evolution of Soybean mosaic virus-G7 molecularly cloned genome in Rsvl-genotype soybean results in emergence of a mutant capable of evading Rsv1mediated recognition. Virology 314:497-509.

Hajimorad, M. R., Eggenberger, A. L. and Hill, J. H. 2005. Loss and gain of elicitor function of Soybean mosaic virus G7 provoking Rsv1-mediated lethal systemic hypersensitive response maps to P3. J. Virol. 79:1215-1222.

Hajimorad, M. R., Eggenberger, A. L., and Hill, J. H. 2006. Strain-specific P3 of Soybean mosaic virus elicits Rsv1-mediated extreme resistance, but absence of P3 elicitor function alone is insufficient for virulence on Rsvl-genotype soybean. Virology 345:156-166.

Hajimorad, M. R., Eggenberger, A. L., and Hill, J. H. 2008. Adaptation of Soybean mosaic virus avirulent chimeras containing P3 sequences from 
virulent strains to $R s v 1$-genotype soybeans is mediated by mutations in HC-Pro. Mol. Plant-Microbe Interact. 21:937-946.

Hayes, A. J., Jeong, S. C., Gore, M. A., Yu, Y. G., Buss, G. R., Tolin, S. A., and Saghai Maroof, M. A. 2004. Recombination within a nucleotidebinding-site/leucine-rich-repeat gene cluster produces new variants conditioning resistance to Soybean mosaic virus in soybeans. Genetics 166:493-503.

Hull, R. 2002. Matthew's Plant Virology. Academic Press, New York.

Jayaram, C. H., Hill, J. H., and Miller, W. A. 1992. Complete nucleotide sequences of two Soybean mosaic virus strains differentiated by response of soybean containing the Rsv resistance gene. J. Gen. Virol. 73:2067-2077.

Jenner, C. E., Tomimura, K., Ohshima, K., Hughes, S. L., and Walsh, J. A 2002a. Mutations in Turnip mosaic virus P3 and cylindrical inclusion proteins are separately required to overcome two Brassica napus resistance genes. Virology 300:50-59.

Jenner, C. E., Wang, X., Ponz, F., and Walsh, J. A. 2002b. A fitness cost for Turnip mosaic virus to overcome host resistance. Virus Res. 86:1-6.

Krause-Sakate, R. E., Redondo, E., Richard-Forget, F., Jadao, A. S.,
Houvenaghel, M. C., German-Retana, S., Pavan, M. A., Candresse, T., Zerbini, F. M., and Gall, O. Le. 2005. Molecular mapping of the viral determinants of systemic wilting induced by a Lettuce mosaic virus (LMV) isolate in some lettuce cultivars. Virus Res. 109:175-180.

Mackey, D., Holt, B. F., III, Wiig, A., and Dangl, J. L. 2002. RIN4 interacts with Pseudomonas syringae type III effector molecules and is required for RPM1-mediated resistance in Arabidopsis. Cell 108:743-754.

Rodríguez-Cerezo, E., and Shaw, J. G. 1991. Two newly detected nonstructural viral proteins in potyvirus-infected cells. Virology 185:572579.

Rost, B., Yachdav, G. and Liu, J. 2004. The PredictProtein server. Nucleic Acids Res. 32:321-326.

Sambrook, J., and Russell, D. W. 2001. Molecular Cloning: A Laboratory Manual, 3rd ed. Cold Spring Harbor Laboratory Press, Cold Spring Harbor, NY, U.S.A.

Wang L., Eggenberger, A., Hill, J., and Bogdanove, A. J. 2006. Pseudomonas syringae effector $A v r B$ confers soybean cultivar-specific avirulence on Soybean mosaic virus adapted for transgene expression but effector AvrPto does not. Mol. Plant-Microbe Interact. 19:304-312. 